\title{
DUST DISTRIBUTION AND TEMPERATURE IN THE MAGELLANIC CLOUDS INTERSTELLAR MEDIUM
}

\author{
M. SAUVAGE, L. VIGROUX \\ Service d'Astrophysique \\ C.E.N. Saclay \\ 91191 Gif-sur-Yvette \\ France
}

\begin{abstract}
This review deals with the various aspects of interstellar dust in the Magellanic Clouds (MCs). Dust properties can be traced from interstellar absorption, with an emphasis on UV properties, and from infrared emission. Thanks to IRAS, most of the recent developments in this field have been found in the infrared. The low resolution of IRAS was, in fact, well suited for MC mapping and these observations offer a unique opportunity to study the dust properties in various conditions of the InterStellar Radiation Field (ISRF) and of chemical abundances. The proximity of the MCs allows a direct study of the link between the stellar population and the dust properties via the ISRF. On the other hand, the comparison of IRAS data in the MCs and in the Galaxy allows us to study the dust composition for metallicity varying by a factor of 10 . From these data and the previous results on UV absorption, it emerges that if the gas-todust ratio changes with metallicity, there is also a variation in the relative abundances of the dust components. In this review, we will also discuss how our knowledge of the MCs will be used to prepare for observations of more distant galaxies, with the next generation of space observatories such as the Hubble Space Telescope and the Infrared Space Observatory.
\end{abstract}

\section{Introduction}

To understand the dust component of the MCs, it is useful to remember a general characteristic of their InterStellar Medium (ISM), i.e. the low abundance of heavy elements: as shown by Dufour (1984), and Dennefeld (1989) in HII regions, the metallicity is about $Z_{\Theta} / 4$ in the $L M C$ and $Z_{\odot} / 10$ in the SMC. It is obvious that the abundances of such elements as Silicon, Oxygen and Carbon (the elementary bricks of every dust model (Léger \& Puget 1984, Mathis \& Whiffen 1989) are of primary importance when the dust component is investigated.

Since the launch of IRAS, our knowledge of the dust itself has grown more precise. It is now quite clear that the two privileged wavelength domains for its study are the UV, where the dust gets most of its energy, and the IR where this energy is re-radiated. The exact mechanisms for absorption and emission clearly depend on the size of the dust grain and different processes are sampled according to the sampled wavelength. Controversy is still raging on the nature of the different dust components and a compared study of dust in the MCs and in the Galaxy, added to the determination of the UV InterStellar Radiation Field (ISRF) can help shed light on this matter.

We shall briefly review what is known from extinction measurements; this type of study is very useful for placing constraints on the nature of the dust but is useless when aiming at its distribution (limited number of sampled lines-of-sight). The latter is more easily seen in emission, i.e. in the infrared, and we shall discuss this matter extensively. 


\section{Extinction measurements}

Since the basis of the method is to compare a reddened star with a non-reddened one, the problem of finding the right match is crucial. In the Clouds, this problem gets more complicated since spectral types are poorly known. Furthermore, $\mathrm{E}(\mathrm{B}-\mathrm{V})$ is small in the MCs which will magnify the mismatch errors. To date, samples consist of about 20 stars in each Cloud. In such small samples, the significance of the mean seems uncertain, more so since we expect very different physical conditions in regions such as the LMC Bar or 30 Dor. Yet the compared behaviour of the mean curves provides indeed some evidences on major changes in the dust composition.

\subsection{MEAN EXTINCTION CURVES}

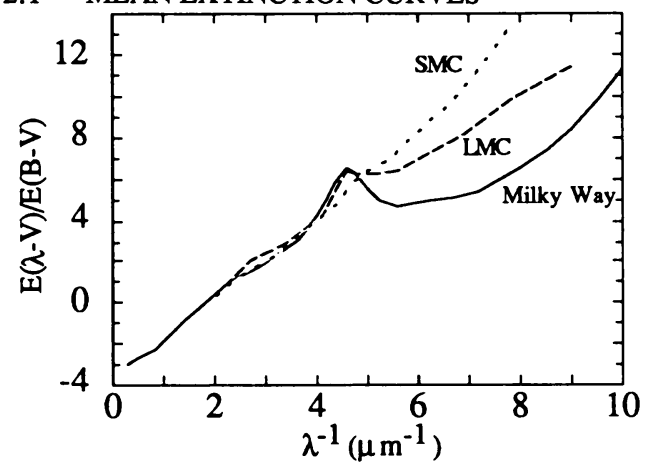

Figure 1. Extinction curves for the Galaxy, the LMC and the SMC (see text for references).

Fig. 1 shows the Galactic mean extinction curve (Savage \& Mathis 1979) from the near IR to the far UV where most of the interesting modifications will occur. On the same figure are plotted results from Koornneef and Code (1981) for the LMC and Bouchet et al. (1985) for the SMC. A noticeable feature in this figure is the similarity of all the curves from the NIR to the near UV, implying a constant $R=A_{V} / E(B-V)$ in the three Galaxies. But the most striking one is the apparent continuity of changes in the UV from the Galaxy to the LMC and the SMC: the $2175 \AA$ bump gets weaker as metallicity decreases, while the FUV rise gets stronger.

The constancy of the NIR to visible part implies that the metallicity is not the major parameter ruling the abundance of big $(0.1$ to $0.01 \mu \mathrm{m})$ grains generally thought to cause this extinction (Désert, Boulanger, Puget 1990, hereafter DBP). On the contrary, changes affecting the UV part of the curve reveal some dramatic evolution in the dust composition. A straight-forward interpretation of the observed trend would lead to the following picture: grains responsible for the FUV rise are more abundant in the LMC than in the Galaxy and even more so in the SMC. On the other hand, the disappearnce of the $2175 \AA$ bump implies that the carriers of this absorption feature are less and less abundant as one goes from the Galaxy to the LMC and the SMC. As this feature is generally associated with carbonaceous material (Mathis \& Whiffen 1989, Lequeux 1988, 1989, Bouchet et al. 1985), this seems to be coherent with the under-abundance of Carbon in the Clouds (Dufour 1984). Yet, as noted by Lequeux (1988), this abundance is only the gaseous one and the solid one may indeed be higher. Association of the FUV rise with PAH macro-molecules as proposed by DBP is problematic since it implies that PAH are over-abundant in the SMC, a conclusion not supported by the infrared emission of the Clouds.

Finally, we point to the results of Fitzpatrick $(1985,1986)$ for the LMC and Lequeux (1988) for the SMC who shoed that there is a wide scatter in extinction properties inside the Clouds. This 
variety is to be expected since the MCs are irregular galaxies, and should be reflected in their IR properties.

\subsection{GAS-TO-DUST RATIO}

From extinction measurements, an estimation of the gas-to-dust ratio can be performed through $\mathrm{N}_{\mathrm{H}} / \mathrm{E}(\mathrm{B}-\mathrm{V})$, provided that one is able to supply $\mathrm{N}_{\mathrm{H}}$. Ratios computed by several authors (Bohlin et al. 1978 for the Galaxy, Fitzpatrick 1985 for the LMC and Bouchet et al. 1985 for the SMC) yield a clear tendency for $\mathrm{N}_{\mathrm{H}} / \mathrm{E}(\mathrm{B}-\mathrm{V})$ to increase as the metallicity decreases: $4.8 \times 10^{21} \mathrm{~cm}^{-2} \mathrm{mag}^{-1}$ for the Galaxy, $2.4 \times 10^{22} \mathrm{~cm}^{-2} \mathrm{mag}^{-1}$ for the LMC and $5.2 \times 10^{22} \mathrm{~cm}^{-2} \mathrm{mag}^{-1}$ for the SMC. This can be easily understood as the heavy elements' abundances should rule, for a given mass of gas, the abundance of dust.

In summary, the relative abundances of dust components inside the dust phase are modified as the metallicity decreases although the way this is done is not yet clear. The infrared emission, coupled with the determination of the UV ISRF will put some strong constraints on the explanations one can put forward.

\section{Dust seen in emission}

Given the expected temperature for dust in the ISM (30K), the thermal emission will peak somewhere around $100 \mu \mathrm{m}$, making the IR the vest wavelength to observe dust. This is reinforced by the fact that emission is practically always optically thin so that it is roughly proportional to the column density. But the main problem we are faced with in the IR is that the observed emission is the combined effect of the dust-mass distribution, its chemical composition and the ISRF (the energy source). A first step toward the resolution of this problem is to get the ISRF. This can be done in the MCs since sources of this field are mainly young stars $\left(<10^{7}\right.$ yrs $)$ that can be surveyed from the ground. Below, we shall make use of this survey to compare with the IR properties (for details see Sauvage et al. 1990).

The complexity of the process that leads to IR emission makes it difficult to speak of dust distribution or temperature; thus we shall rather discuss the distrubtion of the IR emission and IR colours and make the link to the former quantities when possible.

\subsection{DISTRIBUTION OF IR EMISSION}

The IR maps (Fig. 2) used here are part of the Large Optical Galaxies Catalogue (Rice et al. 1988) and consist of co-added IRAS scans.

The general distribution of the IR emission, as traced by $60 \mu \mathrm{m}$, resembles the distribution of other tracers of the ISM (when available). In particular, the $60 \mu \mathrm{m}$ LMC map is very well correlated with the CO survey of Cohen et al. (1988). A concentration of HI emission (Rohlfs et al. 1984) coincides with the elongated component southward of 30 Dor while the Shapley III constellation is seen as a hole in both the IR and HI. The lack of similar data for the SMC prevents such analysis.

Another remarkable characteristic of the IR maps is the association of nearly every infrared peak with a catalogued HII region. This association with star-forming or young regions is confirmed by the comparison with the age distribution in the Clouds. Regions dominated by very young stars are located on infrared peaks (30 Dor, N11, NGC 346) while older ones are linked to infrared holes (Shapley III). 

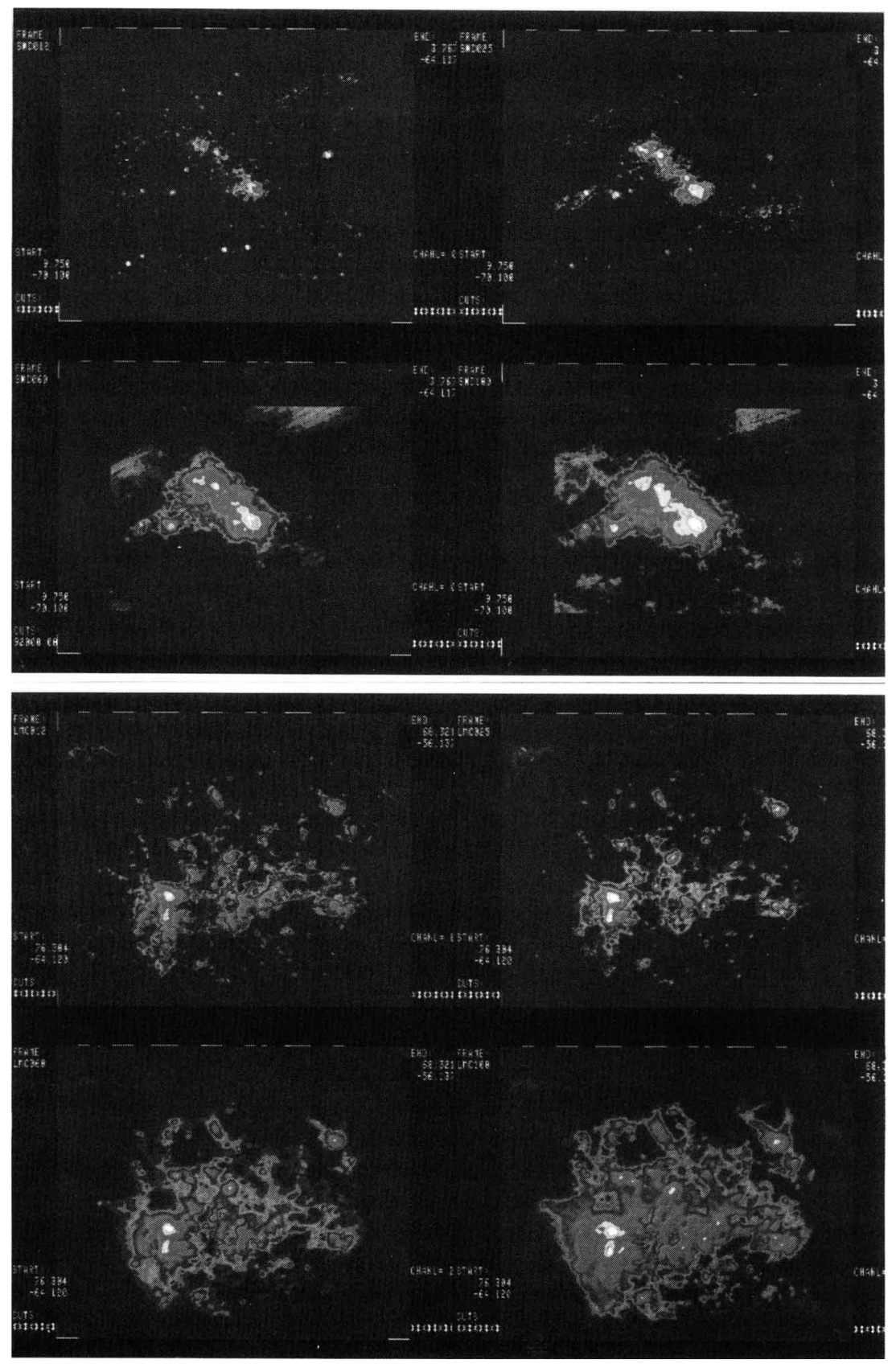

Figure 2. IRAS co-added maps of the SMC (top) and LMC (bottom). From left to right and top to bottom are the $12,25,60$ and $100 \mu \mathrm{m}$ maps. Low and high cuts have been adjusted to give the same dynamic range in all images. Note pre-eminence of 30 Dor in LMC and the weak $12 \mu \mathrm{m}$ image of SMC. 
Note, also, the very close similarity of the distribution of the emission at all four wavelengths (IRAS $12,25,60,100 \mu$ ) suggesting that all the dust components are physically associated down to scales of a few $10 \mathrm{pc}$. This ceases to be true on smaller scales as revealed by Galactic nebulae observations (Boulanger et al. 1988, 1990, Cox et al. 1990) showing that on these scales only do the physical processes (UV field, shocks) become strong enough to destroy some dust components. The similarity of the $12 \mu \mathrm{m}$ map with the other wavelength favours a general ISM origin (PAHs as proposed by Léger \& Puget 1984) for this emission rather than a photospheric cr circumstellar one.

As a last remark on the IR emission distribution, we point to the $12 \mu \mathrm{m}$ emission in the SMC which looks rather weak compared to the LMC. Although work in progress (Viallefond private communication) is revealing extended emission we will show below that there is a real effect independent of sensitivity problems.

\subsection{INFRARED COLOURS}
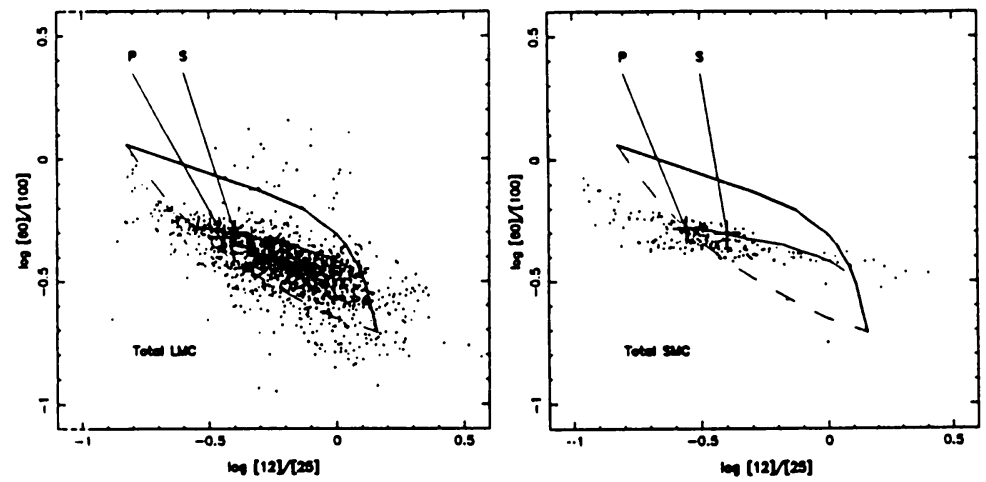

Figure 3. Standard colour-colour diagrams of the MCs. The upper curve is the result of Désert's (1986) model while the lower one is the derived phenomenological mode of Hélou (1986). Crosses show the mean colours from Schwering $(1988, S)$ and Sauvage et al. $(1990, \mathrm{P})$.

The general trend in the "standard" (Hélou 1986) colour-colour diagram ([12]/[25] v. [60]/[100]) shows an anti-correlation of these two colours. This is interpreted using a model where 60 and $100 \mu \mathrm{m}$ are mainly thermal radiation from big grains $(0.1$ to $0.01 \mu \mathrm{m})$ while $12 \mu \mathrm{m}$ is non-thermal emission from very small grains (PAHs; for details on this model, see Désert 1986). Such a colour sequence is also present in the MCs (Fig. 3). Furthermore, correlation of the IR colours with the stellar age distribution (Fig. 4) confirms the interpretation of the colour sequence as a temperature sequence, the hottest part being the high [60]/[100] and low [12]/[25] zone. The similarity of the colour distribution shows that the same model can be roughly applied to the infrared emission in the MCs. Yet there are interesting differences in these diagrams. First, there is practically no SMC point in the cool end of the diagram. This would mean that quiet cirrus-like regions do not exist in the SMC; but it is more likely to result from sensitivity limits since these regions are low surface brightness ones in all four bands. Second, the phenomenological model of Hélou (1986) provides a reasonable fit to the LMC data but fails to describe the SMC. Quantitatively, for active regions, the SMC extends toward lower values of [12]/[25] than the LMC. This is most easily seen in the age-colour diagrams: there is practically no overlap of the two distributions. 
Finally, the infrared colours of the MCs reveal that both [12]/[25] and [25]/[60] are lower in the SMC than in the LMC while [60]/[100] is higher. Correlation of the $[25] /[60]$ colour with stellar age shows that it is dominated by thermal process and so there is a discrepancy between the temperature predicted for the two Clouds by the thermal end of the IR spectrum. All the previous results are discussed in detail in Sauvage et al. (1990).
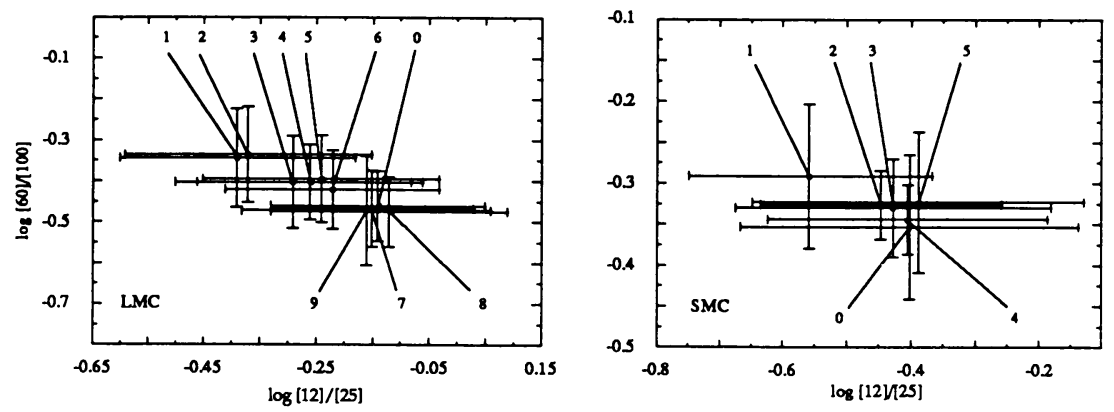

Figure 4. Correlation of the infrared colours with the stellar age. Note that the scales are different so that there is no overlap. Numbers indicate increasing stellar age group; 0 refers to "no stars".

3.2.1 The $12 \mu \mathrm{m}$ deficiency. A better way to characterize this effect is to use the ratio of $\mathrm{vf}_{\mathrm{v}}(12 \mu \mathrm{m}) / \mathrm{FIR}$ which, in the current model, represents the relative abundance of very small grains compared to the large ones. Both in scatter diagrams and with integrated colours, there is a general decrease of $\mathrm{vf}_{\mathrm{v}}(12 \mu \mathrm{m}) / \mathrm{FIR}$ as one goes from the Galaxy to the LMC and the SMC. We interpret this trend as resulting from the decrease of the very small grain abundance. Two effects can lead to this decrease: an increase in the UV field combined with the higher gas-to-dust ratio in the SMC could destroy these grains more easily, or the lower metallicity may simply prevent their formation. But the UV field is equivalent in both Clouds (Lequeux 1989) and the higher gas-to-dust ratio is unable to explain the general trend (Sauvage et al. 1990).

Therefore, the metallicity appears as the right factor. Indeed, in the sample of Irregular and Blue Compact Galaxies of Vigroux et al. (1987) and Kunth and Sèvre (1985) $\mathrm{vf}_{\mathrm{v}}(12 \mu \mathrm{m}) / \mathrm{FIR}$ does increase with the $\mathrm{O} / \mathrm{H}$ abundance ratio (Fig. 5). Thus we conclude that the metallicity limits the grain formation and is able to act selectively on the dust components.

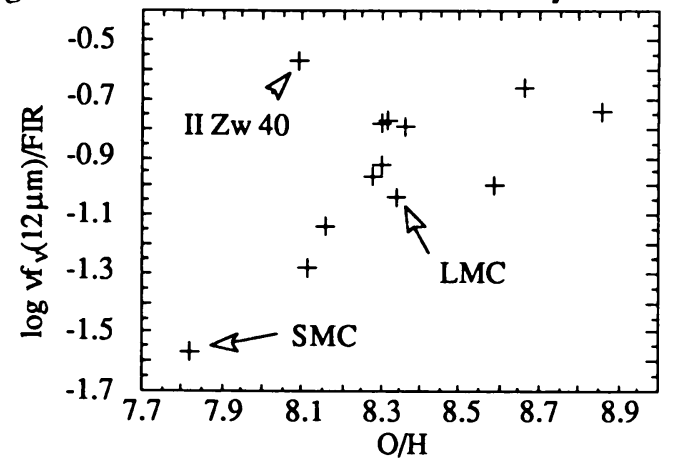

Figure 5. Variation of the small/big grains abundance ratio with $\mathrm{O} / \mathrm{H}$. II $\mathrm{Zw} 40$ seems discrepant but this galaxy is located very close to the Galactic plane, and so its $100 \mu \mathrm{m}$ flux is likely to be contaminated by Galactic Cirrus. 
3.2.2 The $25 \mu \mathrm{m}$ band and the size distribution. The $25 \mu \mathrm{m}$ band cannot be simply the result of the PAH emission from one side and the tail of the modified black-body radiation of big grains from the other. Indeed, the [12]/[25] intensity ratio for PAH only is about 1 (Boulanger et al. 1988, Cox et al. 1990) so that added to the colour temperature of big grains only $10 \%$ at most of the collected emission can be accounted for. Furthermore, such a model would lead to a contradition in the predicted temperature of the thermalized part of the dust phase.

A simple way to recover agreement is to postulate the existence of another thermalized population whose peak wavelength would be located between 25 and $60 \mu \mathrm{m}$ (to obtain the thermal behaviour of this colour). By solving for the two temperatures one gets the SMC slightly hotter than the LMC in both temperatures (cold component with T 27K and hot component with T 55K with a much larger spread in the hot component). This could be obtained physically (see DBP or Puget \& Léger 1989, for instance) with a grain population of size intermediate between the smallest big grains $(0.01 \mu \mathrm{m})$ and the PAHs $(\sim 1 \mathrm{~nm})$.

Thus the SMC appears hotter in the infrared than the LMC. An explanation for this is not so straightforward since in the sample of irregular galaxies no trend for their getting hotter as metallicity decreases, is seen.

\section{Conclusions and prospects}

The dust composition is not homogeneous in the Clouds though there is a general trend to see the carriers of the $2175 \AA$ bump disappear while grains responsible for the FUV rise become more abundant. Yet the infrared emission shows that the very small grains emitting at $12 \mu \mathrm{m}$ are less abundant, preventing any association of this population with the FUV feature.

Metallicity plays a key role in the dust phase: it is able, selectively, to prevent the formation of the different species. In particular, very small grains seem to be more difficult to form in low metallicity environments. Concerning the gas-to-dust ratio, though one sees that it is decreasing with the metallicity for the Galaxy, the LMC and the SMC, it is not clear whether metallicity really is the driving parameter. Indeed, were it the case, one should get, for a constant UV ISRF, hotter and hotter colour temperatures. Data in our possession suggest that it is not the case, but we clearly lack strong observational evidence.

This will be provided by the coming generation of space observatories: the Hubble Space Telescope will create access to the stellar population of a larger sample of galaxies, allowing for the determination of their UV field, while the Infrared Space Observatory will perform imaging and detailed spectroscopy on the same objects. Thus it will be possible to acquire further understanding of the dust phase in galaxies; in particular, there may be answers as to the number and nature of the distinct dust components, the link between the dust and other phases of the ISM, and how the UV ISRF, the gas-to-dust ratio and the metallicity interact to produce and destroy the dust phase.

\section{Acknowledgments}

All the analysis of the infrared data was done with T.X.Thuan. We express our thanks for his valuable help. 


\section{References}

Bohlin, R.C., Savage, B.D., Drake, J.F. (1978), Astrophys. J. 224, 132.

Bouchet, P., Lequeux, J., Maurice, E., Prévot, L., Prévot-Burnichon, M.L. (1985), Astron. Astrophys. 149, 330.

Boulanger, F., Beichman, C., Désert, F.X., Hélou, G., Pérault, M., Ryter, C. (1988), Astrophys. J. 332, 328.

Boulanger, F., Falgarone, E. Puget, J.L., Hélou, G. (1990), Astrophys. J. (in press).

Cohen, R.S., Dame, T.M., Garay, G., Montani, J., Rubio, M., Thaddeus, P. (1988), Astrophys. J. 331, L95.

Cox, P., Deharveng, L., Leene, A. (1990), Astron. Astrophys. 230, 181.

Dennefeld, M. (1989), Recent developments of Magellanic Cloud Research, K.S. de Boer, G. Stasinska, F. Spite (eds.), (Observatoire de Paris: Paris) p.107.

Désert, F.X. (1986), Light on Dark Matter, F.P. Israel (ed.) (Reidel: Dordrecht) p.353.

Désert, F.X. , Boulanger, F., Puget, J.L. (1990), Astron. Astrophys. (in press).

Dufour, R.J. (1984), IAU Symp 135 Structure and Evolution of the Magellanic Clouds, S. Van den Bergh, K.S. de Boer (eds.) (Reidel: Dordrecht) p.353.

Fitzpatrick, E.L. (1985), Astrophys. J. 299, 219.

Fitzpatrick, E.L. (1986), Astron. J. 92, 1068.

Hélou, G. (1986), Astrophys. J. 311, L33.

Koornneef, J., Code, A.D. (1981), Astrophys. J. 247, 860.

Kunth, D., Sévre, F. (1985), Star-Forming Dwarf Galaxies and Related Objects, D. Kunth, T.X. Thuan, J.T.T. Van (eds.) (Institut d'Astrophysique: Paris) p.331.

Léger, A., Puget, J.L. (1984), Astron. Astrophys. 137, L5.

Lequeux, J. (1988), Dust in the Universe, M.E. Bailey, D.A. Williams (eds.) (Cambridge University Press: Cambridge) p.449.

Lequeux, J. (1989), Recent developments of Magellanic Cloud Research, K.S. de Boer, G. Stasinska, F. Spite (eds.), (Observatoire de Paris: Paris) p.119.

Mathis, J.S., Whiffen, G. (1989), Astrophys. J. 341, 808.

Puget, J.L., Léger, A. (1989), Ann. Rev. Astron. Astrophys. 27, 161.

Rice, W., Lonsdale, C.J., Soifer, B.T., Neugebauer, G., Kopan, E.L., Lloyd, L.A., de Jong, T., Habing, H.J. (1988), Astrophys. J. Suppl. Ser. 68, 91.

Rohlfs, K., Kreitzchmann, J., Stegman, B.C., Feitzinger, J.V. (1984), Astron. Astrophys. 137, 343.

Sauvage, M., Thuan, T.X., Vigroux, L. (1990), Astron. Astrophys. (in press).

Savage, B.D., Mathis, J.S. (1979), Ann. Rev. Astron. Astrophys. 17, 73.

Schwering, P.B.W. (1988), PhD. thesis, Leiden.

Vigroux, L., Stasinska, G., Comte, G. (1987), Astron. Astrophys. 172, 15. 